\title{
RAPID DESIGN OF R/C COLUMNS USING MACHINE LEARNING TECHNIQUES
}

\author{
Vassilis K. Papanikolaou ${ }^{1}$, and Aristotelis E. Charalampakis ${ }^{2}$ \\ ${ }^{1}$ School of Civil Engineering \\ Aristotle University of Thessaloniki \\ Thessaloniki, 54124, Greece \\ billy@civil.auth.gr \\ ${ }^{2}$ Department of Civil Engineering \\ University of West Attica \\ Athens, 12244, Greece \\ achar@uniwa.gr
}

\begin{abstract}
The development of Machine Learning, which is deemed to be the path to Artificial Intelligence, has changed tremendously the way many computationally intensive tasks are treated nowadays. Regarding the design of $R / C$ columns and bridge piers, the results of a recent project which proposes a number of design functions are examined and discussed in this work. Both rectangular and circular as well as solid and hollow sections are treated. The proposed design functions are naturally immune to numerical instabilities and achieve more than adequate accuracy for design. They are also, by nature, orders of magnitude faster than any design algorithm based on iterative equilibrium procedures. The error estimation for each function is described in detail based on extensive test sets. Certain method pitfalls, which were encountered and successfully treated, are also discussed.
\end{abstract}

Keywords: Machine Learning; Artificial Neural Networks; Big Data; Reinforced Concrete; Design; Columns; Bridge piers. 


\section{INTRODUCTION}

Reinforced concrete (R/C) vertical members (e.g., columns, bridge piers, structural walls) are generally subjected to a combined action comprising two perpendicular planes of flexure (biaxial bending) and a usually compressive axial load. The magnitude and ratio between these three actions $\left(\mathrm{N}, \mathrm{M}_{\mathrm{x}}, \mathrm{M}_{\mathrm{y}}\right)$ mainly depends on the stiffness and position of the member within the structure and the applied loading combination (e.g., gravity, seismic, wind). The design of critical sections for these vertical members, i.e., the calculation of required reinforcement, is normally carried out either by use of traditional design charts (e.g., [1]) or by more rigorous algorithmic solutions that require modern computational resources (e.g., [2,3]). Numerous repetitions of the above design process are usually required at structural level; therefore, its computational efficiency is of prime importance.

In this paper, a fundamentally different approach to the design of $\mathrm{R} / \mathrm{C}$ vertical members is suggested; instead of applying design data (e.g., section dimensions, material properties, etc.) and computational rules (e.g., stress integration, section equilibrium, etc.) to produce answers to the design problem (i.e., the required reinforcement), the exact inverse procedure is applied; using a robust section analysis algorithm, an extensive set (tens of millions) of sample solutions to the problem is first compiled. Then, large Artificial Neural Networks (ANNs) are trained to predict the solution, i.e., the required reinforcement. The obvious reward of this approach is that the forward-propagation can be transformed into closed-form design functions which are by orders of magnitude faster than any iterative schemes. They are also immune to any numerical instability issues.

\section{MACHINE LEARNING}

Machine learning (ML) provides the technical background of data mining, i.e., extraction of information from large datasets, focused on predictions based on known data properties [4]. This is not something new; simple regression analyses based on experimental data can be considered to be early ML applications in engineering. However, regarding the $\mathrm{R} / \mathrm{C}$ design problem investigated herein, such attempts have not gained much recognition due to the limited amount of experimental data, which may lead to overfitting and eventually inaccurate predictions. Contrarily, the proposed approach operates directly on a huge amount of precalculated results which are based on structural mechanics principles and Code regulations [e.g., 5]. It is true that the structure of the produced design functions is obscure; yet, when utilized as a black-box, their results are easily verifiable by comparing them to the results of classic iterative computational methods.

The objective of this work is to produce the simplest possible algorithm (function) that yields an acceptable level of accuracy for practical design purposes. In this context, the initial goal for the calculation of mechanical reinforcement ratio $(\omega)$ was to produce a maximum absolute error of 0.01 , corresponding to an actual reinforcement area error as low as $0.3 \%$ (in a typical R/C column design). Experimentation with various ML implementations showed that only relatively large Artificial Neural Networks (ANNs) have the adequate complexity to address the high nonlinearities inherent in the data. Moreover, data overfitting can be avoided effectively by using extremely large training sets. These training sets were evenly spaced within a standard range of values for each design parameter. Also, additional engineered parameters were applied to assist the learning process. In order to validate of the applied procedure, new testing sets, unknown to the trained ANN, of double the size of their training counterparts were also compiled. The final measure to ensure the robustness of the proposed method was to apply auxiliary ANN-based functions to screen out unreasonable user input that may cause false output due to extrapolation. 


\section{ANN TRAINING \& TESTING}

For training and testing the ANN-based design functions, extensive sets were precalculated using a robust derivative-free algorithm for section analysis under biaxial bending and axial load [3]. Four section types were considered (Rectangular / Circular, Solid / Hollow) using normalized input parameters, similar to traditional design charts [1]. Two notable novelties were the inclusion of the actual concrete cover (c) instead of cover ratios and the consideration of uniformly distributed reinforcement along the section perimeter (constant As per unit length - subscript ' $d$ '), additional to the standard equal amount per side (As/4 - subscript 'e') for rectangular sections. Fig. 1 shows the considered section parameters for the various section types.
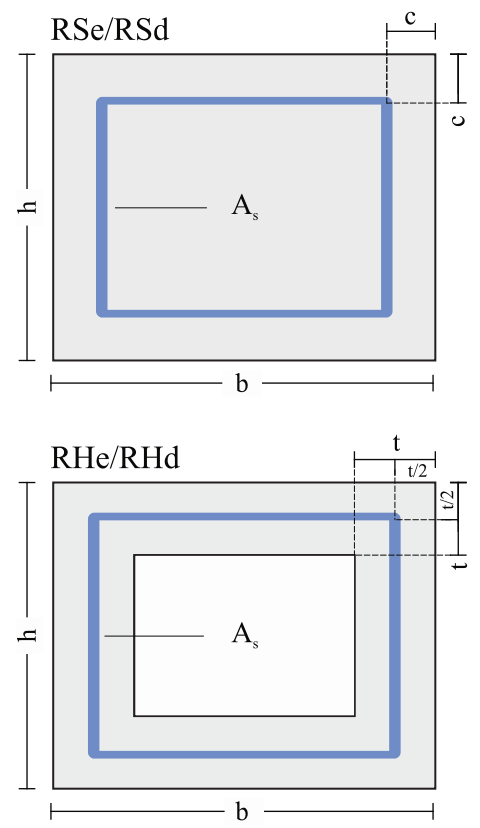
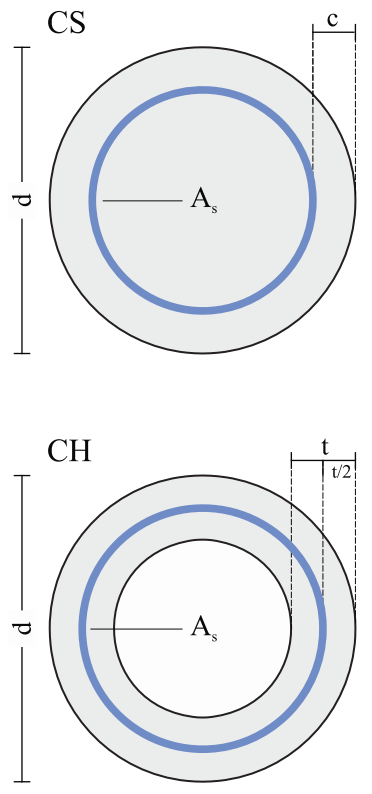

Fig. 1: Section design parameters
C12-C50 $\left(\varepsilon_{\mathrm{c} 2}=2.0 \%, \varepsilon_{\mathrm{cu} 2}=3.5 \%\right.$, $\left.\mathrm{n}=2\right)$ B500C $\left(\mathrm{f}_{\mathrm{yk}}=500 \mathrm{MPa}, \gamma_{\mathrm{s}}=1.15, \mathrm{k}=1.0, \varepsilon_{\mathrm{ud}}=\infty\right)$

$\begin{array}{ll}\omega & \text { mechanical reinforcement ratio } \\ \mathrm{f}_{\mathrm{cd}} & \text { concrete design strength } \\ \mathrm{f}_{\mathrm{yd}} & \text { steel design strength }\left(\mathrm{f}_{\mathrm{yk}} / \gamma_{\mathrm{s}}\right) \\ \gamma_{\mathrm{s}} & \text { steel partial safety factor } \\ \mathrm{k} & \text { steel hardening factor } \\ \varepsilon_{\mathrm{c} 2} & \text { concrete strain at max stress } \\ \varepsilon_{\mathrm{cu} 2} & \text { concrete crushing strain } \\ \varepsilon_{\mathrm{ud}} & \text { steel crushing strain } \\ \mathrm{n} & \text { polynomial order } \\ \mathrm{A}_{\mathrm{c}} & \text { concrete area } \\ \mathrm{A}_{\mathrm{s}} & \text { reinforcement area } \\ \mathrm{b} & \text { section width } \\ \mathrm{h} & \text { section height } \\ \mathrm{d} & \text { section diameter } \\ \mathrm{c} & \text { cover thickness } \\ \mathrm{t} & \text { hollow section thickness } \\ \mathrm{N}_{\mathrm{d}} & \text { axial load } \\ \nu & \text { normalized axial load } \\ \mathrm{M}_{\mathrm{xd}}, \mathrm{M}_{\mathrm{yd}}, \mathrm{M}_{\mathrm{d}} & \text { bending moments } \\ \mu_{\mathrm{x}}, \mu_{\mathrm{y}}, \mu & \text { normalized bending moments }\end{array}$

Normal strength concrete $(\mathrm{C} 12 / 15$ to $\mathrm{C} 50 / 60)$ with parabolic $(\mathrm{n}=2)$ stress-strain relationship and standard B500C steel grade (no hardening, no strain limit) was considered, according to the provisions of the latest draft of Eurocode 2 (EN1992-1-1 [6]). Typical ranges for section parameters were (i) a normalized axial load ( $v$ ) from -0.6 to 0.1 (compression negative), (ii) rectangular section aspect ratios $(\mathrm{b} / \mathrm{h}$ ) from 1.0 (square) to 2.0, (iii) hollow section width to size ( $\mathrm{t} / \mathrm{b}$ or $\mathrm{t} / \mathrm{d}$ ) from 0.05 to 2.0 , (iv) concrete cover to section size ratios (c/b or $\mathrm{c} / \mathrm{d}$ ) from 0.01 to 0.15 and $(\mathrm{v})$ mechanical reinforcement ratios from zero (no reinforcement) to 0.7 , which is deemed a sufficient upper limit for practical design purposes. More details on the ANN setup and the compilation of training/testing sets can be found in [6]. Table 1 shows the set sizes (number of instances) that were finally processed in the present ANN implementation.

\begin{tabular}{llllll} 
Solid section & Training & Test & Hollow section & Training & Test \\
\hline CS & 88,394 & 177,675 & CH & 176,823 & 355,385 \\
RSe & $37,009,460$ & $75,131,088$ & RHe & $41,637,616$ & $84,032,253$ \\
RSd & $37,009,459$ & $75,131,295$ & RHd & $41,637,623$ & $84,032,269$
\end{tabular}

Table 1: Training/test set sizes 


\section{RESULTS}

The training procedure ran for several days on a modern PC with 128 GB of RAM and produced six closed form design functions for the considered section types. All analyses presented herein have been carried out using Python/TensorFlow. Table 2 shows a detailed overview of these functions, together with the ANN configuration (nodes in the input/inner/output layers). Moreover, it includes the mean absolute error (MAE) and the maximum positive and negative errors (MPE/MNE), in terms of calculated mechanical reinforcement ratio $(\omega)$, when tested against independent sets of double the training size. It is apparent that in all cases, the maximum error is well below the initial target of $\omega=0.01$.

\begin{tabular}{lllll} 
Section & Function & ANN size & MAE & MPE/MNE \\
\hline CS & $\omega=f(\mathrm{c} / \mathrm{d}, v, \mu)$ & $3-15-15-1$ & 0.000125 & $0.000947 /-0.001123$ \\
CH & $\omega=f(\mathrm{~d} / \mathrm{d}, \mathrm{v}, \mu)$ & $3-15-15-1$ & 0.000117 & $0.000902 /-0.000858$ \\
RSe & $\omega=f\left(\mathrm{~b} / \mathrm{h}, \mathrm{c} / \mathrm{b}, v, \mu_{\mathrm{x}}, \mu_{\mathrm{y}}\right)$ & $9-35-35-1$ & 0.000186 & $0.006404 /-0.004423$ \\
RSd & $\omega=f\left(\mathrm{~b} / \mathrm{h}, \mathrm{c} / \mathrm{b}, v, \mu_{\mathrm{x}}, \mu_{\mathrm{y}}\right)$ & $9-35-35-1$ & 0.000207 & $0.005233 /-0.005694$ \\
RHe & $\omega=f\left(\mathrm{~b} / \mathrm{h}, \mathrm{t} / \mathrm{b}, \nu, \mu_{\mathrm{x}}, \mu_{\mathrm{y}}\right)$ & $9-35-35-1$ & 0.000170 & $0.004691 /-0.003494$ \\
RHd & $\omega=f\left(\mathrm{~b} / \mathrm{h}, \mathrm{t} / \mathrm{b}, \nu, \mu_{\mathrm{x}}, \mu_{\mathrm{y}}\right)$ & $9-35-35-1$ & 0.000203 & $0.005037 /-0.006202$
\end{tabular}

Table 2: Design functions and ANN performance

The above functions are provided in various programming languages $(\mathrm{CH}, \mathrm{C}++$, Delphi, Fortran, Java, MATLAB, Python, VB.NET, VBA) in the following GitHub repository:

\section{https://github.com/rclab-auth/Project-Omega}

Fig. 2 shows the typical source code of one of these functions, together with a provided spreadsheet that facilitates their use in everyday practice.

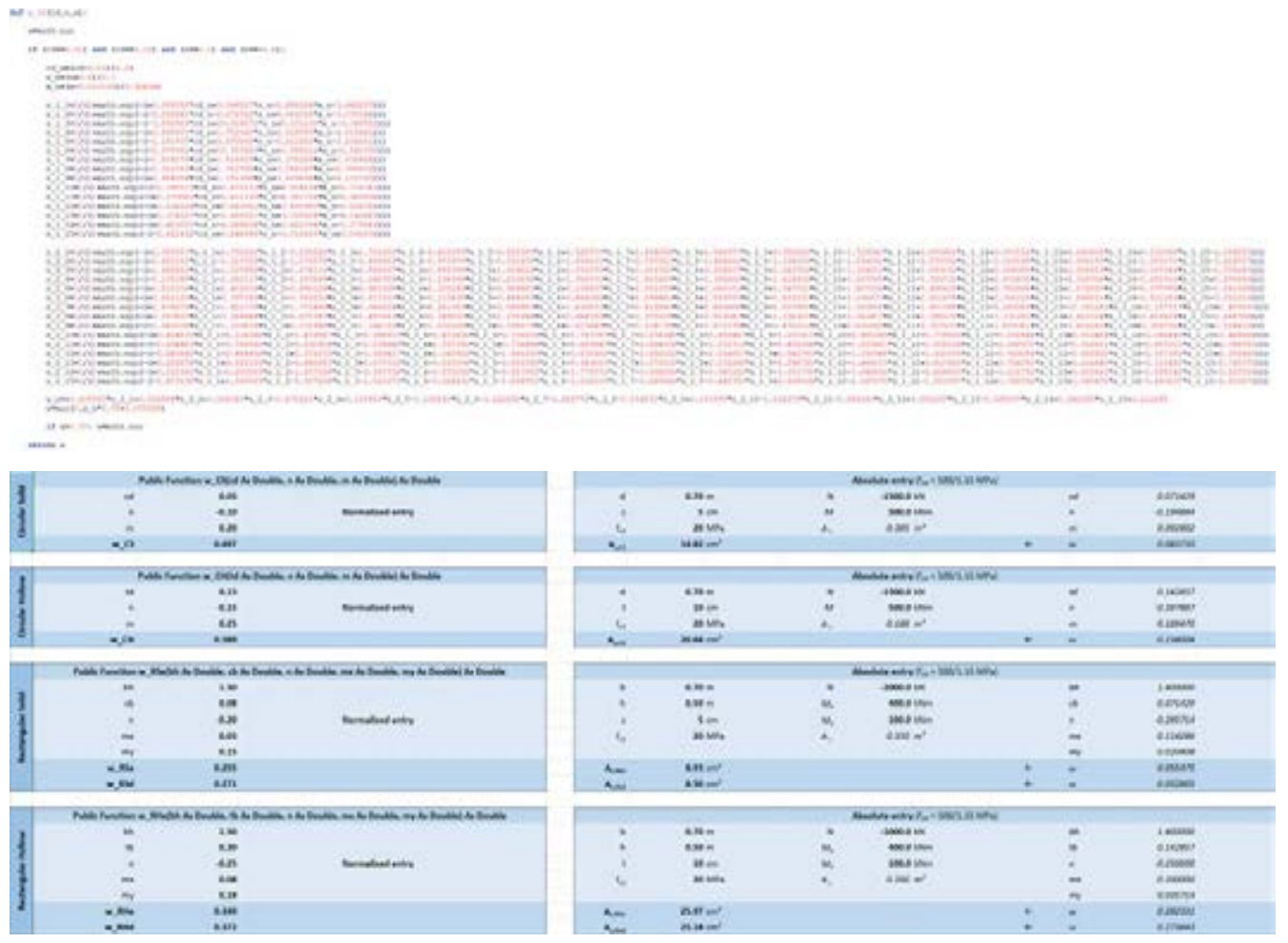

Fig. 2: Function code (Python) and spreadsheet format (Excel) 


\section{ISSUES / VALIDATION / BENCHMARK}

In order to prevent invalid user input, all input variables were limited to the aforementioned ranges on which the respective functions were trained for; however, this could not be applied for normalized bending moments $\left(\mu_{\mathrm{x}}, \mu_{\mathrm{y}}\right)$, since their limit values were not known in advance, depending mainly on the axial load level $(v)$. This led to unpredictable function behavior for unreasonably high user input for bending moments, which, due to the high nonlinearity of design functions, may wrongly predict values for $(\omega)$ in the valid range of $[0,0.7]$ (extrapolation issues, Fig. 3a). To remedy this problem, suitable boundary functions were introduced using small ANNs trained on a fixed value of $\omega=0.75$, in order to successfully control invalid user input (Fig. 3b). A detailed description of this procedure can be found in [6].

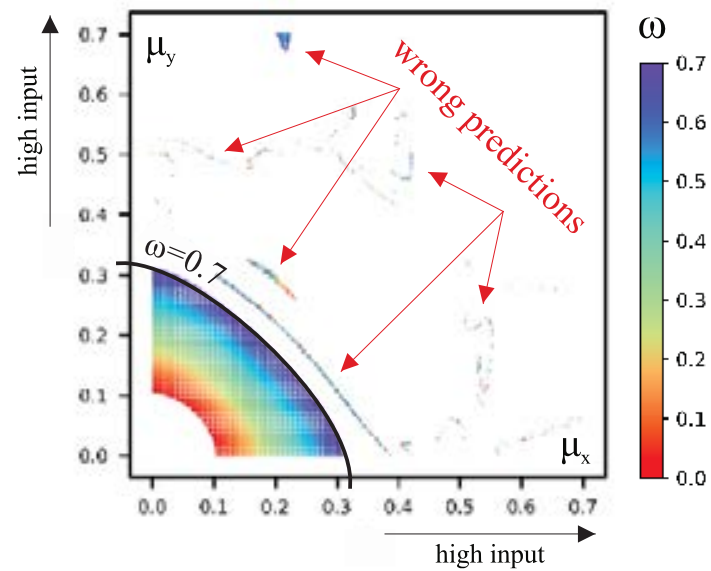

(a)

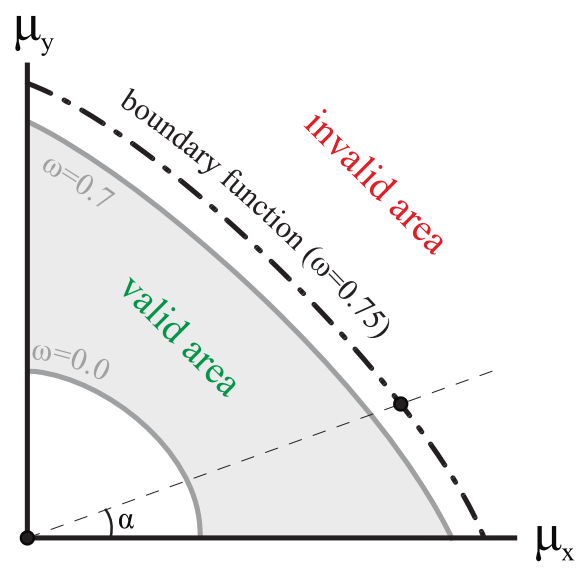

(b)

Fig. 3 Extrapolation issues and boundary functions

The accuracy of the suggested design functions was further tested against well-established uniaxial and biaxial design charts [7,1] using millions of moment and axial load combinations that were directly overlaid on charts together with the calculated mechanical reinforcement ratio $(\omega)$, as shown in Fig. 4. In all cases, correlation was excellent.

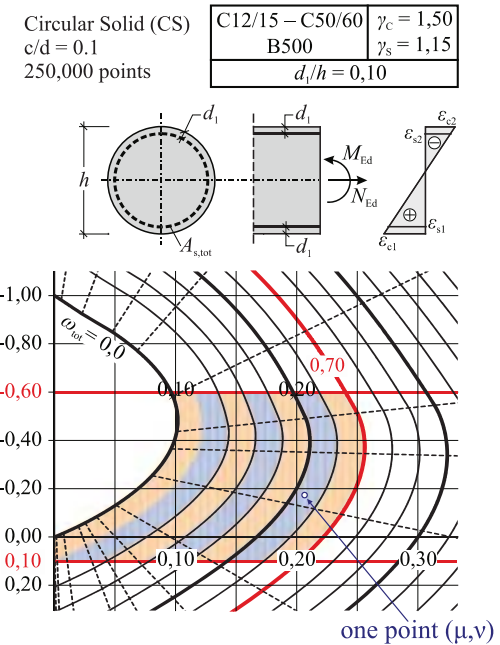

(a)

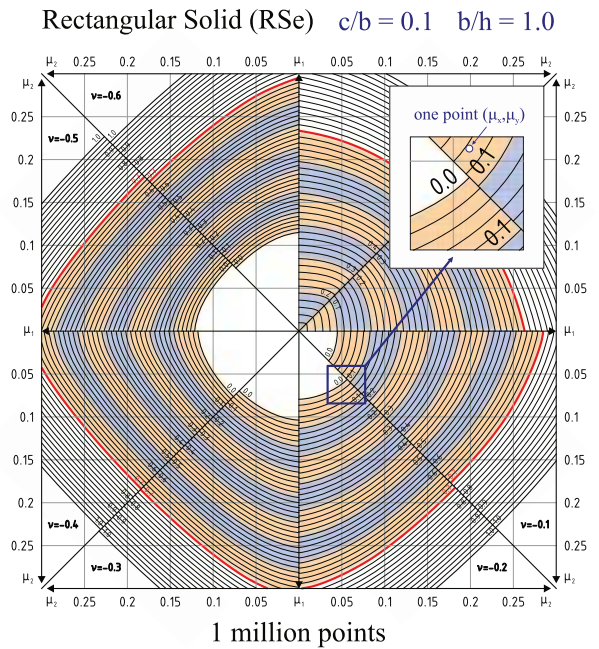

(b)

Fig. 4 Design function tests against (a) uniaxial [7] and (b) biaxial design charts [1] 
Finally, for evaluating the computational performance of the proposed design functions, one million rectangular solid section configurations were calculated, and execution time was compared against an identical run with a classic fast biaxial design iterative algorithm [8]. It was measured that the execution time for the ML-based functions was about 120 times faster ( $5 \mathrm{~s}$ compared to $600 \mathrm{~s}$ on an average PC). Since the proposed design functions produce closed form (i.e., non-iterative) solutions, it is claimed that these will always outperform by a large margin any iterative design method based on stress integration.

\section{CONCLUSIONS}

In this paper, novel closed-form design functions are proposed for the rapid and accurate design of $\mathrm{R} / \mathrm{C}$ columns and bridge piers under biaxial bending with axial load, based on relatively large Artificial Neural Networks. The use of these functions outperforms any known iterative design methods by orders of magnitude and it is immune to any numerical instabilities. Moreover, improved features not available in classic design charts, such as constant concrete cover thickness, hollow rectangular sections and constant reinforcement per unit length along the perimeter have been implemented.

All functions are available in a public repository in multiple programming languages, ready to be integrated into design software with minimal effort. It is believed that similar ANN-based regression procedures can effectively substitute traditional programming approaches in many engineering problems, providing the desired accuracy with unprecedented computational performance.

\section{REFERENCES}

[1] Papanikolaou VK, Sextos AG. Design charts for rectangular R/C columns under biaxial bending: A historical review toward a Eurocode-2 compliant update. Eng Struct 2016;115:196-206. doi:10.1016/j.engstruct.2016.02.033.

[2] Charalampakis AE, Koumousis VK. Ultimate strength analysis of composite sections under biaxial bending and axial load. Adv Eng Softw 2008;39:923-36. doi:10.1016/j.advengsoft.2008.01.007.

[3] Papanikolaou VK, Analysis of arbitrary composite sections in biaxial bending and axial load. Comput Struct 2012;98-99:33-54. doi:10.1016/j.compstruc.2012.02.004.

[4] Witten IH, Frank E, Hall MA, Pal CJ. Data mining : practical machine learning tools and techniques. 4th ed. Morgan Kaufmann; 2016.

[5] CEN Eurocode 2: Design of concrete structures - Part 1-1: general rules and rules for buildings 2004:EN 1992-1-1.

[6] Charalampakis, A.E. and Papanikolaou, V.K. Machine learning design of R/C columns. Eng Struct 2021;226:111412.doi:10.1016/j.engstruct.2020.111412.

[7] Holschemacher K, Müller T, Lobisch F. Bemessungshilfsmittel für Betonbauteile nach Eurocode 2. Weinheim, Germany: Wiley-VCH Verlag GmbH \& Co. KGaA; 2012. doi:10.1002/9783433602102

[8] Penelis GG. Analytical investigation of the biaxial bending problem of R/C sections using plasticity theory (in Greek). Aristotle University of Thessaloniki, 1969. 